Lun 02

SURGICAL RESECTION OF LUNG CANCER: PROGNOSTIC SIGNIFICANCE OF TMM STAGING

L.Sunder-Plassmann (a.G.), H. Dienemann (a.G.), G. Heberer

Lung cancer is the most frequent malignant disease in the male population, accounting for $37 \%$ of certified causes of death from all malignant diseases (M. Bates: Bronchial carcinoma, Springer Berlin 1984). There is still general agreement in the literature that surgical resection of all non small cell types still carries the best prognosis providing an overall five year survival rate of approx. 25\%. Marked progress in surgery and anesthesiology has decreased operative mortality of lobectory and pneunonectomy from $15-20 \%$ down to $2-5 \%$. However it has been pointed out repeatedly that al.1 propress in surgical techniques - has not been able to improve overall survival figures within the past 30 years: Longterm sucess of surgery is extremly dependant upon TM staging: 5 year survival in $\mathrm{T1}$ NO tumors is reported up to $90 \%$, whereas $\mathrm{TX} N 2$ tumors carry a poor prognosis with 5 years survival of approx. 13\% (N. Martini 1982). In our own group of 464 patient, resected for lung cancer from 1979 to 1985 only $13 \%$ were 11 tumors whereas in $64 \%$ the tumor had increased to $\mathrm{T} 2$ and in $23 \%$ to $\mathrm{T} 3$ stages. Infiltration of mediastinal lymphnodes (LN) which carries the worst prognosis was found in almost one fifth of all resections, perioperative mortality was $2 \%$ for Iobectomy and pneumonectomy. Hi lar and mediastinal. LN staging was carried out in every patient, radical mediastinal $\mathrm{LN}$ dissection perfomed whenever lymphnodes where found to be enlarged. In contrast to general beleave only $40 \%$ of enlarged $L N(<20 \mathrm{~m})$ were infiltrated by carcinoma whereas $60 \%$ were enlarged by nonspecific lymphadenitis.

From our experience we conclude that:

1. Early diagnosis of bronchial cancer has to be improved, since late results of surgery are excellent in early bronchial cancer and operative mortality is low $(\sim 2 \%)$.

2. Mediastinal lymphnode enlargenent per se does not preclude curative resection. $L N$ staging and radical dissection however is a must.

3. Postoperative radiation of positive mediastinal lymphnodes needs further documentation.

Chirurgische Klinik und PolikLinik, Klinikum Großhadern,

Marchioninistraße 15, D-8000 Minchen 70

\section{Lun 03}

DIFFERENTIAL EXPRESSION OF BIOMARKERS AND IN VITRO GROWTH PROPERTIES OF ESTABLISHED HUMAN LUNG CANCER CELLL LINES

G. Bepler, G. Jaques, K. Neumann, C. Gropp and K. Havemann

The treatment of small cell lung cancer (SCLC) and of non small cell lung cancer (NSCLC) has failed to further improve survival rates during recent years indicating the need to study the biology of lung cancer in detail in order to establish new treatment modalities that are more specifically adapted to the properties of lung cancer. To accomplish this task, we have established 7 SCLC and 4 non-SCLC cell Iines from body cavity effusions, bone marrow aspirates, and solid tumor lesions of lung cancer patients undergoing diagnostic or treatment procedures. All SCLC cell lines (designated SCLC-16H, $-21 \mathrm{H},-22 \mathrm{H},-24 \mathrm{H},-86 \mathrm{MI}$, $-86 \mathrm{M} 2,-203 \mathrm{H})$ grew as floating cell aggregates in serum-supplemented medium (RPMI $1640+10 \%$ FBS) and with equal growth rates in serum-free medium (RPMI + selenium, insulin, transferrin). Population doubling times range $\bar{c}$ from $45-200 \mathrm{hr}$, and all cell lines formed colonies in soft agarose with an efficieny of $0.06-3.4 \%$. Nude mouse xenografts that developed with a latent period of 4-8 weeks after subcutaneous inoculation of $2 \times 10^{6}$ cells showed typical small cell histology of the intermediate cell type, and in one instance a mixed small cell/large cell histology was found. U1trastructural analysis revealed dense core granules in all SCLC cell lines. The levels of the neuroendocrine system markers L-dopa decarboxylase, neuron-specific enolase, and the total creatine kinase activity as well as the level of its BB-isoenzyme clearly distinguished SCLC cell lines from non-SCLC cell lines (designated EPLC-32M4, EPLC-65M2, ICLC-103H, MSTO-211H) with marker levels being elevated in SCLC cell lines only. Detectable levels of bombesin, the 14 amino acid amphibian peptide recently suggested to function as an autocrine growth factor in SCLC, were found by means of a polyclonal double antibody radioimmunoassay in $2 / 6$ SCLC and $0 / 4$ nonSCLC cell lines. Flow cytometric DNA analysis revealed near diploid indices in all SCLC cell lines and hyperploid indices in non-SCLC cell lines. These well characterized continuously growing lung cancer cell lines may provide an easy basis to further investigate the biology of lung cancer.

Zentrum für Innere Medizin der Philipps-Universität, Abt. Hämatologie/ Onkologie, Balcingerstrasse, D-3550 Marburg
Lun 04

EGF BINDING SITES ARE PRESENT IN NON-SMALL CELL LUNG CANCER CELL LINES AND THE URTNARY BLADDFR CARCTNOMA CTLT LTNE 5637

M. Häder, M. Rotsch, G. Bepler, P. Kiefer and K. Havemann

Today oncogene research is closely linked to growth factor research because cancer cells are thought to produce and respond to their awn growth factors (autocrine secretion). The discovery of sequence homologies between the erb- $B$ oncogene product and the internal and membrane part of the EGF receptor (Downward et al., Nature 307, 521, 1984) suggests a correlation between oncogenic stimulation and growth regulatory mechanisms of cells. The EGF receptor may play a central role in cancer growth control. Thus we examined EGF binding sites in the urinary bladder carcinoma cell line 5637 and in different non-small cell lung cancer cell lines. Time course experiments of ${ }^{125} \mathrm{~J}-\mathrm{EGF}$ binding at various incubation temperatures $\left(4^{\circ} \mathrm{C}, 24^{\circ} \mathrm{C}, 37^{\circ} \mathrm{C}\right)$ showed an increased EGF binđing with increasing temperatures and incubation periods. EGF binding decreased, however, after long incubation periods at high temperatures which can be explained by receptor internalization and degradation. Displacement experiments đemonstrated competition of labeled EGF with unlabeled EGF. The binding of EGF was saturable. Non-specific binding as calculated from the differences between samples with anc without an excess amount of unlabeled EGF was approximately 1.6 \%. Scatchard plot analysis revealed a maximum binding of $1430 \mathrm{fmol}$ EGF/mg protein by the urinary bladder carcinoma cell line 5637 (130000 molecules EGF/cel1) and $845 \mathrm{fmol}$ EGF/mg protein by the non-small cell lung cancer cell line EPLC-65M2. The apparent dissociation constant was $3 \times 10^{-9} \mathrm{M}$. The maximum anount of binding sites and the dissociation constants were in agree ment with the values found in other cell lines (Carpenter et al., J. Biol. Chem. 250, 4297, 1975; Parker et al., J. Biol. Chen. 259, 9906, 1984; Pitzpatrick et al., Cancer Res. 44, 3442, 1984). These results demonstrate the presence of saturable high affinity EGF binding sites on non-small cell lung cancer celi lines and a urinary bladder carcinoma cell line. To further characterize the EGF binding sites, inmunohistochemistry and competition studies of EGF receptors with monoclonal antibodies are necessary, in particular with respect to the possible growth regulation of cancer cells by EGF/EGF receptor interaction.

Zentrum für Innere Medizin der Philipps-Universität, Abt. Hämatologie/ Onkologie, Baldingerstrasse, D-3550 Marburg

\section{Lun 05}

THE VALUE OF THE BILATERAL ILIAC CRESI NEEDLE BIOPSY FOR THE PRETHERAPEUTIC TUMOR STAGING OF LUNG CANCER - RESULTS FROM A TNM FIELD STUDY C.Manegold,H.Bülzebruck, B.Krempien, P.Drings

Our study is to clarify whether the bilateral iliac crest biopsy could be of value for confirming bone marrow involvement(BMI) in bronchogenic carcinoma at the time of diagnosis. 243 patients with histologically confirmed lung cancer were biopsied after having given written consent( 221 men, 22 women). The median age was 59 years. $33 \%$ of the patients had a SCCL, and $67 \%$ a NSCCL. Undecalcified bone sections were used for examination. Sole criterion for proof of BMI was the presence of tumor cells. The median surface area of the bone marrow sections examined per patient was $54 \mathrm{~mm}^{2}$. There was no difference between biopsies with and without BMI.7 of the 243 patients (3\%) showed a BMI in the biopsy. These were exclusively cases with $\operatorname{SCCL}(7 / 80=9 \%)$. $3 / 7$ patients exhibited BMI only on one side.All 7 patients with BMI already had distant metastases, large primary tumors, and 1 mph, node involvement. In conclusion: In early stage

NSCCL BMI rarely occurs in the biopsy. It would thus appear that bone marrow biopsy should not be recommended as a routine pretherapeutic procedere. The SCCL shows the highest BMI rate of al1 bronchogenic carcinomas. Its presence can be most easily demonstrated in advanced stages. It is extremely rare that BMI is the only proof of a tumor generalization Under these circumstances we cannot recommend the bone marrow biopsy as a routine measure even for early stages of SCCI. Nevertheless, the needle point biopsy on the iliac crest is still the appropriate method to clarify BMI. Bilateral biopsy is recommended. Medizinische Universitätsklinik, Bergheimer Str.58, D - 6900 Heidelberg 